\title{
Evaluation of Performance for Wireless Sensor Networks Based on Gray Theory
}

\author{
JING Jun-li ${ }^{\text {a }}$, YANG Jie ${ }^{\text {b }}$ \\ ${ }^{a}$ College of Science, Huaihai Industrial College, Lianyungang, China \\ ${ }^{b}$ Departmant of Communication Commandk, Chongqing Communication College, Chongqing, China
}

\begin{abstract}
A performance evaluation method of wireless sensor networks based on gray theory is proposed. Firstly the influence factors of performance are analyzed, and the index set in evaluation of wireless sensor networks' performance is built which include index of key performance and reliable characteristics. Based on AHP and gray theory, a model of evaluation of wireless sensor networks performance is given. Finally the results of example show that the evaluation model is rationality and feasibility.
\end{abstract}

Index Terms: Wireless sensor network(WSN); AHP; gray theory; evaluation of performance.

(C) 2012 Published by MECS Publisher. Selection and/or peer review under responsibility of the Research Association of Modern Education and Computer Science

\section{Introduction}

Wireless sensor networks (WSNs) are special networks comprising a large number of energy-constrained sensor nodes, which are densely deployed throughout physical space for observing surrounding ambient conditions[1]. WSN can be widely used such as military, medical and industrial purpose. And the wireless sensor networks have presented interesting and challenging issues[2]. Inorder to optimize network service performance,designs the new network mechanism, quantification analysis and evaluate WSN is necessary. While the evaluation approach include game theory, AHP, TOPSIS and so on[3-5].

Thereby, suitable evaluation methods should be needed to identify and confirm those wireless sensor networks with more contribution. In this paper, an evaluation index is proposed on the basis of considering different wireless sensor networks performance. The paper uses the gray theory method in the process of evaluation by experts, while the evaluation process is effective.

This paper is organized as follows. In the next section, we describe the influence factors and considerations of performance evaluation forwireless sensor networks. In Section III, based on the gray theory, we present the framework and methods for evaluating performance. Section IV base on the AHP and gray theory, an illustrative example is presented. Finally, conclusion is demonstrated in Section V.

* Corresponding author.

E-mail address: lygzjj1@sina.com, yangjie5581@163.com 


\section{The influence factors of WSN for evaluation}

Since the wireless sensor networks' usability is influenced by its performance immediately, we need to consider how to evaluation of performance for the wirelsss sensor network. The following is the key performance and trust performance of wireless sensor network, as shown in Fig 1[6].

(1)Energy validity

Energy validity of the wireless sensor networks is the number of processable requests, under the condition that the energy of network is limit.

(2) Life cycle

The life cycle of the wireless sensor networks is the time interval which from the network running to unproviding the information for receiver's needs. And the influence factor of life cycle in wireless sensor networks includes hardware and software.

(3) Time delay

The time delay of wireless sensor networks is the time interval which from the request sending to the needs information receiving. And the time delay is related with using closely, which affects the usability and using field of wireless sensor networks.

(4) Sensation precision

The sensation precision of wireless sensor networks is the precision of sensation information from receiver. While the precision of sensor, method of information process, communication protocol of network affect the sensation precision in abundance.

(5) Extendibility

The extendibility of wireless sensor networks is the expansion limit which can be found from the number of sensor, covering field, life cycle, time delay, sensation precision. If the level of extendibility is assignment, the wireless sensor networks should provide the mechanism and method to support the level of extendibility.

(6) Fault tolerance

The sensor of network often useless because of environment or power source exhausts. So the hardware and software have the very strong fault tolerance in the wireless sensor networks, which guarantee high reliability in the system. When hardware or software in the network has breakdown, the system could be automatic control or automatic restructuring.

The trust performance in wireless sensor networks should include four facts[7].

(7) communication characteristic

In the WSN, node behavior includes control order or the transmission of application. When the malicious node maybe discard or distort the data packet, the selfish node possibly discard data packet which needs to retransmit because of saving energy. The node's correspondence behavior has been observed to distinguish malicious node or the selfish node. Also the communication characteristic is one of major performance in trust.

(8) Password mechanism

In the majority of applications, the trust management is supplement method based on the cryptology mechanism, which enhances the network security. At the same time, the password mechanism also becomes one of main consideration factors in many trust evaluation index.

(9) Data application

The data acquisition is main application in WSNs. According to application, sensor node needs to gather data through transmitting data to processing server for information, and the gather data is to be analysis and recorded by the server. In order to reduce the number of information flow, the transmission energy consume, memory demand in the network, data aggregation is applied frequently in upload processing of information, sometimes also be called data fusion. Moreover the trust management usually uses to enhance data fault-tolerant ability, distinguish error message, enhances the upload data the accuracy and so on.

(10) Other performance 
Besides the several primary factors, there are other trust factors to influence network. For instance, a node which has a higher trust value, is chosen to carries out the duty probability higher. Then the electricity consumes quickly, therefore the electrical energy is one of consideration factors in trust value computation. And node's usability is also one of factors in trust value computation.

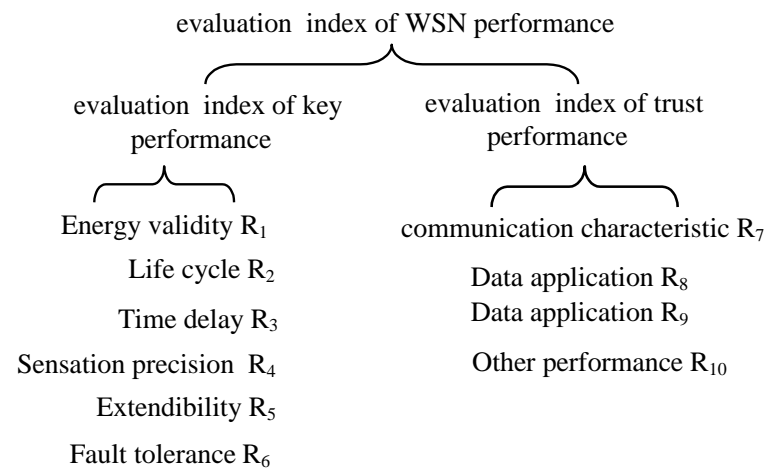

Fig.1. Evaluation index of performance in WSN

\section{Evaluation approach of WSN performance}

In this section, based on evaluation index of performance in WSN, a gray theory-based approach to evaluate performance of WSN is developed, in which a gray judge matrix is used too. Moreover, the judge matrix is given by the whitening function. And the AHP method is applied to determine the weight of evaluation index. And this index in the WSN performance evaluation, such as energy validity, life cycle, time delay and so on, should be calculated to get the judge subjection matrix, using gray stat method. In the following section the step of this evaluation approach is presented.

\section{A. Swatch matrix}

The field of evaluation grade $V=\left(v_{1}, v_{2}, \cdots, v_{c}\right)$ is decided firstly. Where, $v_{g}$ expresses the evaluation grade of number $g$, and $c$ represents the number of evaluation grade. Moreover the evaluation grade of index is distinguish to five grade, which corresponding numerical value is $9,7,5,3,1$. While the numerical value between two grade is $8,6,4,2$. There are $h$ experts to evaluate the performance of wireless sensor networks, the expert give the numerical value $d_{k j}^{h}$ to evaluation index $u_{k j}$. So the original matrix $D_{i}$ of evaluation index is constitution.

$$
D_{i}=\left[\begin{array}{cccc}
d_{11}^{1} & d_{12}^{1} & \cdots & d_{m n}^{1} \\
d_{11}^{2} & d_{12}^{2} & \vdots & d_{m n}^{2} \\
\vdots & \vdots & \vdots & \vdots \\
d_{11}^{p} & d_{12}^{p} & \cdots & d_{m n}^{p}
\end{array}\right]
$$

\section{B. Gray sort of evaluation}

Gray sort of evaluation, as fuzzy and uncertainty, is a variety or concept which could be compared. Since to describe the gray sort, the gray value and whiten weight value should be confirm. However we are unaware of nicety gray value, which include probable range. What is called whiten weight function is presented probability or preference degree of evaluation index, which belong to a certain gray sort in one value range. Based on gray theory, the 5 evaluation gray sort in evaluation of performance for WSN, corresponding to evaluation degree: 
very high,high,moderate,low very,low. That is $\mathrm{e}=1,2,3,4,5$. In view of material analysis for evaluation object, the gray value of gray sort and whiten weight function is given by[8]:

- “gray sort $\mathrm{e}=1$, gray value $\otimes_{1} \in[0,9, \infty)$ ” :

$f_{1}(x)=\left\{\begin{array}{cc}\frac{x}{9} & x \in[0,9] \\ 1 & x \in[9, \infty) \\ 0 & x \in(-\infty, 0)\end{array}\right.$

- "gray sort $\mathrm{e}=2,3,4$, gray value $\oplus_{2} \in[4,7,10], \oplus_{3} \in[2,5,8], \oplus_{4} \in[0,3,6]$, and $\left\{d_{0}, d_{1}, d_{2}\right\}=\{4,7,10\},\{2,5,8\},\{0,3,6\} ":$

$f_{2}(x)=\left\{\begin{array}{cc}\frac{x-d_{0}}{d_{1}-d_{0}} & x \in\left[d_{0}, d_{1}\right] \\ \frac{x-d_{2}}{d_{1}-d_{2}} & x \in\left[d_{1}, d_{2}\right] \\ 0 & x \notin\left[d_{0}, d_{2}\right]\end{array}\right.$

- “gray sort $\mathrm{e}=5$, gray value $\otimes_{5} \in\left[\begin{array}{ll}0,1,3 & 3\end{array}\right.$ ”

$f_{3}(x)=\left\{\begin{array}{cc}1 & x \in[0,1] \\ \frac{x-3}{-2} & x \in[1,3] \\ 0 & x \notin\left[0_{0}, 3\right]\end{array}\right.$

\section{Gray evaluation result}

- Gray evaluation coefficient

By applying the defnition of whiten weight function, we can define the performance evaluation index $u_{k j}$ which belong to eth gray sort. Herein, the gray evaluation coefficient of ith WSN, which belong to eth evaluation gray sort, is defined as $y_{k j e}^{i}$. In addition, the total gray evaluation coefficient is $y_{k j}$. respectively, it is defined as

$y_{k j e}^{i}=\sum_{h=1}^{q} f_{e}\left(d_{k j}^{h}\right)$

$y_{k j}^{i}=\sum_{e=1}^{5} y_{k j e}^{i}$

- Weight vector and evaluation matrix

To index $u_{k j}$, all evaluation expert give the ith wireless sensor network's eth kind of gray evaluation weight $r_{k j e}^{i}$. 
that is

$r_{k j e}^{i}=\frac{y_{k j j_{i e}}^{i}}{y_{k j}^{i}}$

Since the evaluation gray kind have 5 class, the ith wireless sensor network's gray evaluation weight vector is $r_{k j}^{i}$. It is denoted

$r_{k j}^{i}=\left(r_{k j 1}^{i}, r_{k j 2}^{i}, r_{k j 3}^{i}, r_{k j 4}^{i}, r_{k j 5}^{i}\right)$

The matrix of weight vector to evaluation index $u_{k j}$ is $R_{k}^{i}$. It is represented as follow, while the $l$ in (9) is quantity of evaluation index.

$R_{k}^{i}=\left[\begin{array}{c}r_{k 1}^{i} \\ r_{k 2}^{i} \\ \vdots \\ r_{k l}^{i}\end{array}\right]=\left[\begin{array}{cccc}r_{k 11}^{i} & r_{k 12}^{i} & \cdots & r_{k 15}^{i} \\ r_{k 21}^{i} & r_{k 22}^{i} & \cdots & r_{k 25}^{i} \\ \vdots & \vdots & \vdots & \vdots \\ r_{k 11}^{i} & r_{k l 2}^{i} & \cdots & r_{k 15}^{i}\end{array}\right]$

Likewise, we can get all of matrix $R^{i}$ for gray evaluation weight in the ith WSN. It is writen as $R^{i}=\left[\begin{array}{llll}R_{1}^{i} & R_{2}^{i} & \cdots & R_{m}^{i}\end{array}\right]^{T}$

- Gray value of evaluation index

The evaluation index value of the jth evaluation index in ith WSN is given by

$E_{j}^{i}=R_{j}^{i} \bullet Z$

Where, $z$ is class value vector, $z=[9,7,5,3,1]^{T}$

Finally,we can give the Synthesis evaluation for MSN

$P_{i}=\sum_{c=1}^{k} w_{c} \cdot e_{c}^{i}$

Where, $e_{c}^{i}(c=1,2, \cdots, k)$ is evaluation index value of ith WSN, and c is represented quantity of index kind. While $w_{c}$ is represented weight for cth kind index. Repeating the above step, we can obtain the gray evaluation weight matrix and the evaluation index value of other wireless sensor network.

\section{Illustrative example}

In this section, we present an illustrative example to demonstrate the capability of our evaluation approach. In this case, we organize five experts to evaluate 4 types of wireless sensor network. The experts provide comprehensive performance numerical evaluations about the 4 types of wireless sensor network in different circumstance.

\section{Calculation of weights}

In this case, we use AHP method to determine weights. We stratify factors involved in decision making by establishing hierarchy structure which includes target layer, rule layer and project layer to construct a model of hierarchical analysis. We establish judgment matrix, mark low-layer elements dominated by elements of the target layer $U$ with $U_{1}, U_{2}, \cdots, U_{n}$. Decision-makers compare $U_{i}$ and $U_{j}$, and decide which one is more 
important according the target layer. And we get eigenvectors $w=\left(\bar{w}_{1}, \bar{w}_{2}, \cdots \bar{w}_{n}\right)^{T}$ which weights are given by experts. In this case, we get the result through calculating:

$$
\begin{aligned}
& w=\left(w_{1}, w_{2}\right)=(0.667,0.333) \\
& w_{1}=\left(w_{11}, w_{12}, w_{13}, w_{14}, w_{15}, w_{16}\right) \\
& =(0.215,0.143,0.302,0.111,0.105,0.124)
\end{aligned} \quad w_{2}=\left(w_{21}, w_{22}, w_{23}, w_{24}\right)=(0.339,0.233,0.178,0.240)
$$

\section{E. Calculation of grey evaluation matrix}

Analyze evaluation indicators using the white function in the grey theory, construct grey evaluation matrix, we will get the evaluations of each wireless sensor networks.

1) Establishment of the sample matrix

In this case, we take the A-type wireless sensor network as an analysis case : ${ }^{1}$ represent the sample matrix of secondary level indications subject to the first level indications of the I item .

$$
\begin{gathered}
D_{1}^{1}=\left[\begin{array}{l}
d_{11}^{1} \\
d_{12}^{1} \\
d_{13}^{1} \\
d_{14}^{1} \\
d_{15}^{1} \\
d_{16}^{1}
\end{array}\right]^{T}=\left[\begin{array}{llllll}
6 & 7 & 7 & 5 & 7 & 6 \\
8 & 6 & 8 & 7 & 8 & 7 \\
7 & 8 & 7 & 8 & 7 & 7 \\
7 & 6 & 9 & 7 & 7 & 8 \\
7 & 7 & 6 & 6 & 6 & 6
\end{array}\right] \quad D_{2}^{1}=\left[\begin{array}{l}
d_{21}^{1} \\
d_{22}^{1} \\
d_{23}^{1} \\
d_{24}^{1} \\
d_{25}^{1}
\end{array}\right]=\left[\begin{array}{llll}
5 & 7 & 6 & 6 \\
5 & 6 & 8 & 6 \\
7 & 5 & 8 & 7 \\
5 & 6 & 8 & 5 \\
6 & 7 & 5 & 8
\end{array}\right] \\
e=1, y_{111}^{1}=4.333 ; e=2, y_{112}=3.867 ; e=3, y_{113}=0.653 ; e=4, y_{114}=0.000 ; e=5, y_{111}=0.000
\end{gathered}
$$

2) Establishment of grey evaluation coefficient

Calculate grey evaluation coefficient using white weight function and formula(5). We take the A-type wireless sensor network as an analysis case to calculate the grey evaluation coefficient for the index of $u_{11}$.

$$
e=1, y_{111}^{1}=4.333 ; e=2, y_{112}=3.867 ; e=3, y_{113}=0.653 ; e=4, y_{114}=0.000 ; e=5, y_{111}=0.000
$$

Finally, the total grey evaluation coefficient is $y_{11}=\sum_{e=1}^{5} y_{11 e}=8.853$.

3) Calculation of weight vector of grey evaluation and evaluation matrix

Under the evaluation index $u_{11}$, the e class grey evaluation weights of the A-type wireless sensor network which all experts evaluate is marked $r_{11 e}^{1}$.

$$
r_{111}^{1}=0.489, \quad r_{112}^{1}=0.437, r_{113}^{1}=0.074, \quad r_{114}^{1}=0, \quad r_{115}^{1}=0
$$

Grey evaluation weights vector of the index $u_{11}$ of the wireless sensor network A is

$$
r_{11}^{1}=(0.4894,0.4368,0.0738,0.000,0.000)
$$

Calculate the other grey evaluation weights vectors $r_{12}^{1}, r_{13}^{1}, r_{14}^{1}, r_{15}^{1}, r_{16}^{1}$, combine the above and we can get the grey evaluation matrix $R_{1}^{1}$ as follow: 


$$
R_{1}^{1}=\left[\begin{array}{l}
r_{11}^{1} \\
r_{12}^{1} \\
r_{13}^{1} \\
r_{14}^{1} \\
r_{15}^{1} \\
r_{16}^{1}
\end{array}\right]=\left[\begin{array}{lllll}
0.4894 & 0.4368 & 0.0738 & 0.0000 & 0.0000 \\
0.4273 & 0.4718 & 0.1009 & 0.0000 & 0.0000 \\
0.3772 & 0.4521 & 0.1707 & 0.0000 & 0.0000 \\
0.3010 & 0.3801 & 0.2012 & 0.1177 & 0.0000 \\
0.5343 & 0.4024 & 0.0635 & 0.0000 & 0.0000 \\
0.4415 & 0.4122 & 0.1463 & 0.0000 & 0.0000
\end{array}\right]
$$

Repeat the above steps, we can get the grey evaluation matrix of the other secondary level indications subject to the first level indications

$$
R_{2}^{1}=\left[\begin{array}{l}
r_{21}^{1} \\
r_{22}^{1} \\
r_{23}^{1} \\
r_{24}^{1}
\end{array}\right]=\left[\begin{array}{lllll}
0.4286 & 0.3570 & 0.1786 & 0.0357 & 0.0000 \\
0.3667 & 0.3667 & 0.2333 & 0.0333 & 0.0000 \\
0.3298 & 0.3511 & 0.2872 & 0.0319 & 0.0000 \\
0.4512 & 0.4024 & 0.1463 & 0.0000 & 0.0000
\end{array}\right]
$$

4) Calculation of evaluation value of every index by using formula(11)

$$
\begin{aligned}
& E_{1}^{1}=R_{1}^{1} \bullet Z=\left[e_{11}^{1}, e_{12}^{1}, e_{13}^{1}, e_{14}^{1}, e_{15}^{1}, e_{16}^{1}\right] \quad E_{2}^{1}=R_{2}^{1} \bullet Z=\left[e_{21}^{1}, e_{22}^{1}, e_{23}^{1}, e_{24}^{1}\right]=[7.846,7.134,6.958,7.610] \\
& =[7.831,7.653,6.029,7.413,6.729,7.941]
\end{aligned}
$$

5) Calculation of the overall score of the A-type wireless sensor network using formula(12)

Repeat the above steps, we can get the integrated score. The integrated score of the A-type wireless sensor network is 7.268. The integrated scores of the B-type, C-type and D-type are 7.845, 6.935 and 6.646.

\section{5. conclusion}

In this study, we proposed a model to evaluate performance for WSN by using the data of integrated performance evaluation index. The model, based on gray theory, had a better performance than conventional evaluation method. Its advantage was that the whiten ability was much bigger than the linear discriminant evaluation model. Gray theory-based evaluation method which also use the AHP to deside the weight of evaluation index, has a great potential for evaluation of WSN design and practical applications.

\section{Acknowledgment}

This work is supported by National Natural Science Foundation Project (71071173) and Chongqing Soft Sciences Project(CSTC, 2010CE0109).

\section{References}

[1] I.F. Akyildiz, W. Su, "Wireless sensor networks: a survey," Computer Networks vol. 38, 2002, pp. 393422.

[2] Junhua Zhu, Ka-Lok Hung, Brahim Bensaou, et al. "Rate-lifetime tradeoff for reliable communication in wireless sensor networks," Computer Networks vol. 52 ,2008, pp. 25-43.

[3] N. Riaz, M. Ghavami, "Analytical performance evaluation of ultra-wideband multiple access schemes for different wireless sensor network application environments," IET Commun., 2009, Vol. 3, Iss. 9, pp. 1473-1487

[4] I. Chatzigiannakisa, E.Konstantinoub, V. Liagkoua, et al. "Design, Analysis and Performance Evaluation of Group Key Establishment in Wireless Sensor Networks,"Electronic Notes in Theoretical Computer Science vol 171, $2007,17-31$ 
[5] H.B. Chen, C. K. Tse, J.C. Feng, "Performance evaluation of source extraction in wireless sensor networks," Computer Communications vol 31, 2008, pp.3870-3875

[6] Z. A. Eu, H. P. Tan, K.G. Seah, "Design and performance analysis of MAC schemes for Wireless Sensor Networks Powered by Ambient Energy Harvesting," Ad Hoc Networks vol. 9 , 2011, pp. 300-323.

[7] C. G. Zhu, S. C. feng, Z, L. juan, "Design and performance analysis of wireless sensor network location node system for underground mine," Mining Science and Technology vol. 19, 2009, pp. 813-818

[8] C. I. Hsu, Y. H. Wen, "Application of Grey theory and multiobjective programming towards airline network design,"European Journal of Operational Research vol. 127, 2000, pp.44-68 